\title{
Quantum state estimation using weak measurements
}

\author{
Debmalya Das and Arvind \\ Department of Physical Sciences, Indian Institute of Science Education 8 Research (IISER) Mohali, \\ Sector-81, SAS Nagar, Manauli P.O. 140306, Punjab, India *
}

\begin{abstract}
We explore the possibility of using "weak measurements" without "weak value" for quantum state estimation. Since for weak measurements the disturbance caused during each measurement is small, we can rescue and recycle the state, unlike for the case of projective measurements. We use this property of weak measurements and design schemes for quantum state estimation for qubits and for Gaussian states. We show, via numerical simulations, that under certain circumstances, our method can outperform the estimation by projective measurements. It turns out that ensemble size plays an important role and the scheme based on recycling works better for small ensembles.
\end{abstract}

\section{INTRODUCTION}

The quantum superposition principle and the wave function collapse set apart the quantum description of the world from its classical counterpart. As a consequence, in the standard paradigm, the outcome of a single measurement cannot be predicted with certainty and we can only assign probabilities to different outcomes. Since the measurement process disturbs the system and in a projective measurement the state of the system collapses we cannot re-use the state for any further measurement. This necessitates the use of an ensemble of identically prepared states in order to interpret quantum measurements. Ideally, in the large ensemble limit the ensemble average tends to the expectation value of the observable. The question that arises at this point is: what if we are provided with a small ensemble of states and asked to make the best use of it?

Projective measurements require a large coupling between the system and the measuring device. However, if the coupling is made small, we inflict a very small disturbance to the system at the expense of extracting a correspondingly small amount of information ${ }^{1}$. Such measurements are known as weak or unsharp measurements. Such measurements have been introduced in various forms in the past ${ }^{2-8}$. The coupling strength can be tuned to suit the situation and the state can subjected to further measurements to extract more information. Whenever we have a small ensemble, each member can be "weakly" measured more than once with a possibility of extracting more information.

It is that true that all quantum measurements (projective, non-projective, weak etc) can be seen as Positive Operator Valued Measures (POVM). Still it is important to know the details and workings of a measurement scheme. A POVM can also be interpreted as a projective measurement on a larger Hilbert space 1,9,10. For a finite ensemble the upper bound on the amount of information extractable is available ${ }^{11}$. There is always a cost of information extraction from quantum systems in terms of the disturbance caused and that too has has also been explored for the case of weak measurements 12-15.

A recent work by Rozema et. al. suggests some new possibilities that weak measurements can offer with re- spect to Heisenberg's uncertainty relation and the disturbance caused to the state ${ }^{16}$. Oreshkov et. al., in 2005, wrote down a weak measurement POVM and showed that any generalized measurement can be decomposed into a sequence of weak measurements, without using an ancilla 17 . Lundeen et. al. recently came up with a method employing weak values to directly measure the wave function of a quantum system in a pure state 18 and followed it up with a method to measure any general state ${ }^{19}$. For some further developments in this regard see $^{20}$. Unsharp measurements have also been used to make sequential measurements on a single qubit 7 . Other examples of quantum state tomography with weak measurements can be found in 21-23. An approach to perform quantum state tomography using weak measurement POVMs was introduced by Hofmann 24 .

We present in this paper some of our results on state estimation by "weak" measurements 42 . We explore the case of a single qubit and show by explicit simulations how under certain circumstances the weak measurement based state estimation scheme can beat the one based on projective measurements.

\section{WEAK AND UNSHARP MEASUREMENTS}

The measuring apparatus plays a crucial role in quantum measurements; on the one hand it interacts with the quantum system and on the other hand it has classical properties where the outcomes can be read out and recorded. A useful model of this process is available due to von Neumann. Although originally this model was constructed for strong (projective) measurements 25 it has wider applications and can also be applied to weak measurements $\underline{2}-\underline{-2}, 26$.

\section{A. Von Neumann's measurement model for discrete basis}

Consider the measurement of an observable $A$ of a quantum system with eigenvectors $\left\{\left|a_{j}\right\rangle\right\}$ and eigenvalues $\left\{a_{j}\right\}, j=1 \cdots n$. Imagine an apparatus with continuous pointer positions described by a variable $q$ and its 
conjugate variable $p$ such that $[q, p]=i$. The initial state of the measuring device has an initial spread of $\Delta q$ with its Gaussian quantum state $\left|\phi_{i n}\right\rangle$ centered around zero given by

$$
\left|\phi_{i n}\right\rangle=\left(\frac{\kappa}{2 \pi}\right)^{\frac{1}{4}} \int_{-\infty}^{\infty} d q e^{-\frac{\kappa q^{2}}{4}}|q\rangle
$$

where $\kappa=\frac{1}{(\Delta q)^{2}}$ and we have taken $\hbar=1$. The system and the measuring device are made to interact by means of a Hamiltonian,

$$
H=g \delta\left(t-t^{\prime}\right) A \otimes p
$$

where $p$ is the momentum conjugate to the variable $q$, and $g$ is the coupling strength. The Hamiltonian is so chosen that the system and the device get a kick and interact momentarily at $t=t^{\prime}$. Let the initial state $\left|\psi_{i n}\right\rangle$ of the system be written in terms of the eigenstates $\left|a_{1}\right\rangle,\left|a_{2}\right\rangle, \ldots \ldots,\left|a_{n}\right\rangle$ of the operator $A$.

$$
\left|\psi_{\text {in }}\right\rangle=\sum_{i=1}^{n} c_{i}\left|a_{i}\right\rangle
$$

The joint evolution of the system and the measuring device under the coupling Hamiltonian gives an entangled state for $t>t^{\prime}$

$$
\begin{aligned}
& e^{-i \int H d t}\left|\psi_{i n}\right\rangle \otimes\left|\phi_{i n}\right\rangle= \\
& \left(\frac{\kappa}{2 \pi}\right)^{\frac{1}{4}} \sum_{i=1}^{n} \int_{-\infty}^{\infty} d q c_{i} e^{-\frac{\kappa\left(q-g a_{i}\right)^{2}}{4}}\left|a_{i}\right\rangle \otimes|q\rangle
\end{aligned}
$$

The above state consists of a series of Gaussians centered at $g a_{1}, g a_{2}, \cdots, g a_{n}$ for the pointer entangled with corresponding eigenstates $\left|a_{1}\right\rangle,\left|a_{2}\right\rangle \cdots\left|a_{n}\right\rangle$ of the system. At this stage we invoke the fact that the apparatus is classical, because of the fact that only one of the pointer positions actually shows up. This requires the collapse of the wave function which is brought in as something from outside for the classical apparatus! Thus the process is completed with the meter showing only one of the $g a_{i}$ s and the system state collapses into the corresponding eigenstate $\left|a_{i}\right\rangle$. The above analysis holds good only if the Gaussians are well separated or distinct. In contrast, when the Gaussians overlap, which can happen if the coupling strength $g$ is small or the initial spread in the pointer state given by $1 / \kappa$ is large, the scenario changes 2,8,27. This is called the weak or unsharp measurement regime. Weak measurements have been employed in developing recipes for the violation of Bell inequalities 27 and Leggett-Garg inequalities 28 . These have also been recently used to study super-quantum dis$\operatorname{cord}^{29}$.

\section{B. Weak values and post-selection}

In the treatment of weak measurement given by Aharonov, Albert and Vaidmann (AAV), first a subsequent projective measurement of a second observable $B$ is carried out, followed by a post-selection of the output state into one of the eigenstates of the second observable, say $\left|b_{j}\right\rangle$. The weak value of the observable $A$, which was measured in the weak regime, is then defined as

$$
A_{w}=\frac{\left\langle b_{j}|A| \psi_{i n}\right\rangle}{\left\langle b_{j} \mid \psi_{i n}\right\rangle}
$$

When the post-selected state $b_{j}$ is nearly orthogonal to the initial state $\left|\psi_{i n}\right\rangle$ equation[ [5 tells us that the weak value becomes very large, so large that it can lie outside the allowed range of the eigenvalue spectrum $\underline{8,26}$.

The interpretation of weak values is a current topic of research in quantum information theory. Weak values can be complex and the real and the complex parts can be interpreted in terms of the displacements in the position and momentum spaces, respectively, of the measuring device 30 . Weak values have been used to reinterpret the flow of time in quantum mechanics $\underline{31}$ and in the direct measurement of the photon wavefunction $\underline{\underline{18}}$ and in the amplification of small signals $32-34$. Another interesting application of weak values is in connection with quantum Chesire cat experiments 35,36 . There has been criticisms of the method of post-selection as well, namely that the process of post-selection leads to throwing away data and can lead to suboptimal use of information from a measurement. For discussions on the same see $\underline{37} \underline{\underline{39}}$. However, we take a different approach in our work, where we do not do any post-selection i.e. we consider weak measurements without weak values.

\section{Effect of weak and strong measurement on a qubit}

How exactly do we carry out the weak measurement? How much is the effect of a weak measurement on the system? If we carry out weak measurements on all the members of an identically prepared ensemble, what happens to such an ensemble? We illustrate these points by taking an example. Consider a measurement of $\sigma_{z}(z$ component of spin) of a qubit in a fixed quantum state. Following the general prescription given in Equation (2) we write the interaction Hamiltonian as

$$
H=g \delta\left(t-t^{\prime}\right) \sigma_{z} \otimes p
$$

assuming the initial state of the pointer to be the same as that given in Equation (11). The qubit is taken to be in a pure state given by

$$
\left|\psi_{\text {in }}\right\rangle=\cos \frac{\alpha}{2}|0\rangle+\sin \frac{\alpha}{2}|1\rangle
$$

where $|0\rangle$ and $|1\rangle$ are the eigenstates of $\sigma_{z}$ with eigen values +1 and -1 respectively. The combined state of the system and the pointer after the interaction is given 
by taking a special case of Equation (4)

$$
\begin{aligned}
\left|\psi_{\text {out }}\right\rangle= & \left(\frac{\kappa}{2 \pi}\right)^{\frac{1}{4}} \int_{-\infty}^{\infty} d q \cos \frac{\alpha}{2} e^{-\frac{\kappa(q-g)^{2}}{4}}|0\rangle \otimes|q\rangle \\
& +\left(\frac{\kappa}{2 \pi}\right)^{\frac{1}{4}} \int_{-\infty}^{\infty} d q \sin \frac{\alpha}{2} e^{-\frac{\kappa(q+g)^{2}}{4}}|1\rangle \otimes|q\rangle
\end{aligned}
$$

At this stage the apparatus and the system are in an entangled state. An observation of the apparatus will lead to values whose distribution is determined by the above state. It is clear from Equation (8) that the distribution of values of the apparatus is a Gaussian centered around $+g$ for the system input state $|0\rangle$ and is a Gaussian centered around $-g$ for the system input state $|1\rangle$. The width of the Gaussian in each case is given by $1 / \kappa$. By tuning the parameter $\epsilon=\kappa g$ we can change the nature of the measurement in terms of its strength. In our work we have taken $g=1$ so that we have $\epsilon=\kappa$. For large values of $\epsilon$ we have a projective measurement, where the pointer distributions are well separated for the states $|0\rangle$ and $|1\rangle$. Therefore, each reading of the pointer tells us exactly what the state of the system is after the measurement. By repeatedly measuring the same observable we can calculate the expectation value of the observable. The state collapses completely in each measurement and there is no question of re-using these states. However, when the value of $\epsilon$ is small we have two Gaussians that overlap. From an observation of the pointer we do not learn with certainty as to what value to assign to the system spin $z$ component. The pointer positions are weakly correlated with the eigenstates of $\sigma_{z}$. The state is only partially affected and there is a possibility of re-using the state. The effect of the weak measurement in this case can be explicitly calculated and it turns out that there is very little change in the state of the system. The final state of the system can be calculated by taking the state in Equation (4) and then taking a partial trace over the apparatus's degrees of freedom giving us the final mixed state corresponding to the system alone:

$$
\rho_{f}=\frac{1}{2}\left(\begin{array}{cc}
1+\cos \alpha & \left(1-\frac{\epsilon}{8}\right) \sin \alpha \\
\left(1-\frac{\epsilon}{8}\right) \sin \alpha & 1-\cos \alpha
\end{array}\right)
$$

Since $\epsilon$ is small we can conclude that the disturbance caused to the system is also small. Furthermore, the disturbance can be controlled by changing $\epsilon$.

\section{QUANTUM STATE ESTIMATION OF A SINGLE QUBIT}

We now turn to the question of using weak measurements with state recycling for the problem of state estimation of a single qubit.

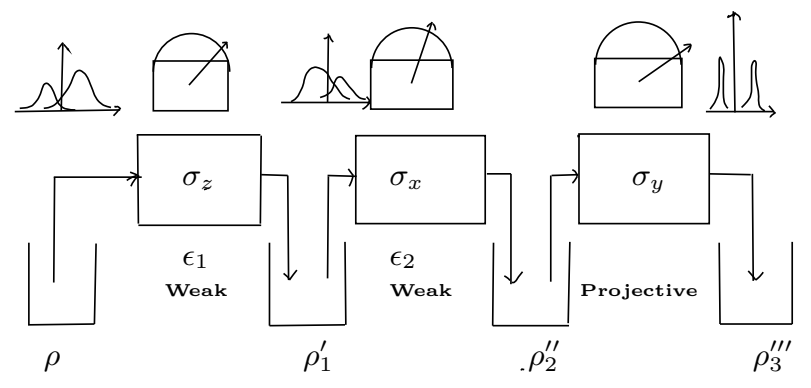

FIG. 1: The schematic diagram of our prescription involving two weak measurements of coupling strengths $\epsilon_{1}$ and $\epsilon_{2}$, allowing state recycling, followed by a projective measurement.

\section{A. The scheme}

In our prescription, we consider a finite size ensemble of pure or mixed states of a qubit. On every member of the ensemble we carry out a $\sigma_{z}$ measurement whose strength is defined by the parameter $\epsilon_{1}$. We record the meter reading in each case and keep the modified states after measurements to obtain a changed ensemble. This new ensemble is now used to measure $\sigma_{x}$ in the same way but with a coupling strength $\epsilon_{2}$. Finally the resultant ensemble is used to carry out projective measurement of $\sigma_{y}$ on its members. The first two measurements are weak while the last measurement is strong or projective. To avoid statistical errors the results are averaged over many runs. The entire process is summarized in Figure 1. For both the weak measurements, consider a regime in which $\epsilon$ is neither too large to make the measurement projective, nor too small, as is done in traditional weak measurements. For such values of $\epsilon$, the two Gaussians, representing the pointer value distributions for the two eigen values of the observable, overlap partially with each other. When there is no overlap, a meter reading unambiguously indicates an outcome and we have a projective measurement. A meter reading corresponding to a point in the overlap region cannot be reliably correlated with the system being in one or the other eigenstate. To reduce this difficulty, let us define a region, midway between the centers of the two Gaussians, of width $2 a$. We call it the discard region, which means that any pointer reading which falls in this region is rejected. For the case where we measure $\sigma_{z}$, all readings where the pointer position is to the right of this region are interpreted as indicating the value of $\sigma_{z}$ to be +1 while the ones on the left of this region are interpreted as -1 . Even when the outcome is discarded, the member of the ensemble is not rejected, but is retained to be re-used for the next measurement. In summary, in this scheme as is shown in Figure 1 we first measure $\sigma_{z}$ weakly, followed by $\sigma_{x}$ which is again measured weakly and last we make a projective measurement of $\sigma_{y}$. The entire simulation is run on identically prepared copies (ensemble size) of the state of interest (pure or mixed). The simulation is repeated many times to avoid statistical errors. 
A general single qubit state is given by

$$
\rho=\rho_{00}|0\rangle\left\langle 0\left|+\rho_{01}\right| 0\right\rangle\left\langle 1\left|+\rho_{10}\right| 1\right\rangle\left\langle 0\left|+\rho_{11}\right| 1\right\rangle\langle 1|
$$

The diagonal elements are known as populations as they give the probabilities with which the states $|0\rangle$ and $|1\rangle$ are present in the mixture. The off-diagonal elements are known as coherences as these contain the phase information of the states $|0\rangle$ and $|1\rangle$. When the state is coupled to a measurement device, as discussed above, the resultant state after unitary evolution for a strength $\epsilon$, is

$$
\begin{aligned}
& \rho^{\prime}=\left(\frac{\epsilon}{2 \pi}\right)^{\frac{1}{2}} \\
& {\left[\int_{-\infty}^{\infty} d q \int_{-\infty}^{\infty} d q^{\prime} \rho_{00} e^{-\frac{\epsilon(q-1)^{2}}{4}} e^{-\frac{\epsilon\left(q^{\prime}-1\right)^{2}}{4}}|0\rangle\langle 0|+\right.} \\
& \int_{-\infty}^{\infty} d q \int_{-\infty}^{\infty} d q^{\prime} \rho_{01} e^{-\frac{\epsilon(q-1)^{2}}{4}} e^{-\frac{\epsilon\left(q^{\prime}+1\right)^{2}}{4}}|0\rangle\langle 1|+ \\
& \int_{-\infty}^{\infty} d q \int_{-\infty}^{\infty} d q^{\prime} \rho_{10} e^{-\frac{\epsilon(q+1)^{2}}{4}} e^{-\frac{\epsilon\left(q^{\prime}-1\right)^{2}}{4}}|1\rangle\langle 0|+ \\
& \left.\int_{-\infty}^{\infty} d q \int_{-\infty}^{\infty} d q^{\prime} \rho_{11} e^{-\frac{\epsilon(q+1)^{2}}{4}} e^{-\frac{\epsilon\left(q^{\prime}+1\right)^{2}}{4}}|1\rangle\langle 1|\right] \\
& \otimes|q\rangle\left\langle q^{\prime}\right|
\end{aligned}
$$

Let us consider taking out a member of the ensemble of system states and then coupling it with the apparatus. Now when the observer notes down the meter reading, she can see a particular reading which depends upon the initial states of the system and the meter and the coupling between the two. Though this process is not well understood and von Neumann's model is silent about this final step of collapse, it can be thought of as the action of the projector $|q\rangle\langle q|$ on the meter state resulting in the meter reading $q$.

The probability density of obtaining the value $q$ for the meter is therefore given by

$$
P(q)=\operatorname{Tr}\left(|q\rangle\langle q| \rho_{M D}\right)
$$

where the reduced density operator for the apparatus or the measuring device (MD) is obtained by taking a partial trace of the state $\rho^{\prime}$ over the system.

$$
\rho_{M D}=\operatorname{Tr}_{\text {system }}\left(\rho^{\prime}\right)
$$

This probability density can now be used to calculate the probabilities of possible outcomes. For example, $P\left(\sigma_{z}=\right.$ 1) can be obtained by integrating the probability density from $+a$ to $\infty$. Thus, the probabilities with which we obtain $+1,-1$ or ambiguous readings while measuring in the $z$-basis are calculated by integrating the above probability densities from $+a$ to $\infty,-\infty$ to $-a$ and $-a$ to $+a$, respectively and are given by

$$
\begin{aligned}
P(|0\rangle) & =\frac{1}{4}\left[(1+z) \operatorname{Erfc} \frac{(-1+a) \sqrt{\epsilon_{1}}}{\sqrt{2}}-(-1+z) \operatorname{Erfc} \frac{(1+a) \sqrt{\epsilon_{1}}}{\sqrt{2}}\right] \\
P(|1\rangle) & =\frac{1}{4}\left[-(-1+z) \operatorname{Erfc} \frac{(-1+a) \sqrt{\epsilon_{1}}}{\sqrt{2}}+(1+z) \operatorname{Erfc} \frac{(1+a) \sqrt{\epsilon_{1}}}{\sqrt{2}}\right] \\
P\left(\operatorname{discard}_{z}\right) & =\frac{1}{2}\left[\operatorname{Erf} \frac{(-1+a) \sqrt{\epsilon_{1}}}{\sqrt{2}}+\operatorname{Erf} \frac{(1+a) \sqrt{\epsilon_{1}}}{\sqrt{2}}\right]
\end{aligned}
$$

Further for the second weak measurement, the input state is the output from the first measurement described by an ensemble $\rho_{1}^{\prime}$. This ensemble is obtained from the state $\rho^{\prime}$ given in Equation (11) by taking a trace over the measuring device (apparatus)

$$
\rho_{1}^{\prime}=\operatorname{Tr}_{M D}\left(\rho^{\prime}\right)
$$

The probabilities with which we obtain the value +1 , -1 or ambiguous readings while measuring in the $\sigma_{x^{-}}$ basis are given by,

$$
\begin{aligned}
P\left(\left|\sigma_{x} ;+\right\rangle\right) & =\frac{1}{4} e^{-\frac{\epsilon_{1}}{2}}\left[\left(-\operatorname{Erf}(-1+a) \sqrt{\frac{\epsilon_{2}}{2}}+\operatorname{Erf}(1+a) \sqrt{\frac{\epsilon_{2}}{2}}\right) x+e^{\frac{\epsilon_{1}}{2}}\left(\operatorname{Erfc}(-1+a) \sqrt{\frac{\epsilon_{2}}{2}}+\operatorname{Erfc}(1+a) \sqrt{\frac{\epsilon_{2}}{2}}\right)\right] \\
P\left(\left|\sigma_{x} ;-\right\rangle\right) & =\frac{1}{4} e^{-\frac{\epsilon_{1}}{2}}\left[\left(\operatorname{Erf}(-1+a) \sqrt{\frac{\epsilon_{2}}{2}}-\operatorname{Erf}(1+a) \sqrt{\frac{\epsilon_{2}}{2}}\right) x+e^{\frac{\epsilon_{1}}{2}}\left(\operatorname{Erfc}(-1+a) \sqrt{\frac{\epsilon_{2}}{2}}+\operatorname{Erfc}(1+a) \sqrt{\frac{\epsilon_{2}}{2}}\right)\right] \\
P\left(\operatorname{discard}_{x}\right) & =\frac{1}{2}\left[\operatorname{Erf} \frac{(-1+a) \sqrt{\epsilon_{1}}}{\sqrt{2}}+\operatorname{Erf} \frac{(1+a) \sqrt{\epsilon_{1}}}{\sqrt{2}}\right]
\end{aligned}
$$


After this measurement if we trace over the second apparatus we obtain the ensemble represented through a density operator $\rho_{2}^{\prime \prime}$. Lastly we perform a regular strong (projective) measurement of $\sigma_{y}$ and the probabilities are given by,

$$
\begin{aligned}
& P\left(\left|\sigma_{y} ;+\right\rangle\right)=\frac{1}{2}\left[1+e^{-\frac{1}{2}\left(\epsilon_{1}+\epsilon_{2}\right)} y\right] \\
& P\left(\left|\sigma_{y} ;-\right\rangle\right)=\frac{1}{2}\left[1-e^{-\frac{1}{2}\left(\epsilon_{1}+\epsilon_{2}\right)} y\right]
\end{aligned}
$$

In the above equations, we have used

$$
\begin{aligned}
\operatorname{Erf}(x) & =\frac{2}{\sqrt{\pi}} \int_{0}^{x} e^{-t^{2}} d t \\
\operatorname{Erfc}(x) & =1-\operatorname{Erf}(x)
\end{aligned}
$$

These measurements when repeated over the entire ensemble give us an estimate of the expectation values of $\sigma_{x}$, $\sigma_{y}$ and $\sigma_{z}$, which in turn help us locate the co-ordinates $(x, y, z)$ of the point inside the Bloch sphere:

$$
\begin{aligned}
& z=\operatorname{Tr}\left(\rho \sigma_{z}\right) \\
& x=\operatorname{Tr}\left(\rho_{1}^{\prime} \sigma_{x}\right) e^{\frac{\epsilon_{1}}{2}} \\
& y=\operatorname{Tr}\left(\rho_{2}^{\prime \prime} \sigma_{y}\right) e^{\frac{1}{2}\left(\epsilon_{1}+\epsilon_{2}\right)}
\end{aligned}
$$

where $\rho, \rho_{1}^{\prime}$ and $\rho_{2}^{\prime \prime}$ denote the initial state of the system and those after the first and second measurements respectively. We note that $\epsilon_{1}$ and $\epsilon_{2}$ appear in Equation (19) because we are interested in the expectation values of $\sigma_{x}, \sigma_{y}$ and $\sigma_{z}$ for the original state $\rho$ of the system. These results are valid only for small values of $\epsilon_{1}$ and $\epsilon_{2}$. In subsequent studies we work with the simplification $\epsilon_{1}=\epsilon_{2}=\epsilon$.

For a scheme based purely on projective measurements, the ensemble is divided into three equal parts and direct measurements of $\sigma_{x}, \sigma_{y}$ and $\sigma_{z}$ are performed independently. This leads to a direct estimate of the expectation values of these operators giving the values of $(x, y, z)$ and hence an estimate of the state. The error in these estimates depends upon the size of the ensemble. We simulate both these schemes and compare the performance of our method with the one based on projective measurements.

We recall that a qubit can be represented as a point in a Bloch sphere ${ }^{9}, 40$.The Bloch sphere is a unit sphere the pure and mixed states of a qubit lying on the surface and inside the sphere, respectively. The state corresponding to the point $(x, y, z)$ is given by

$$
\rho=\frac{1}{2}(I+\vec{n} \cdot \vec{\sigma})
$$

where $\hat{n}=x \hat{x}+y \hat{y}+z \hat{z}$ is a vector with $x=\left\langle\sigma_{x}\right\rangle, y=\left\langle\sigma_{y}\right\rangle$ and $z=\left\langle\sigma_{z}\right\rangle$. The pure states correspond to the case when the point lies on the surface and in that case $\vec{n}$ is a unit vector. The expectation values of $\sigma_{x}, \sigma_{y}$ and $\sigma_{z}$ serve as a direct means to calculate the values of $(x, y, z)$. Therefore, to carry out state estimation of a given state of (a)

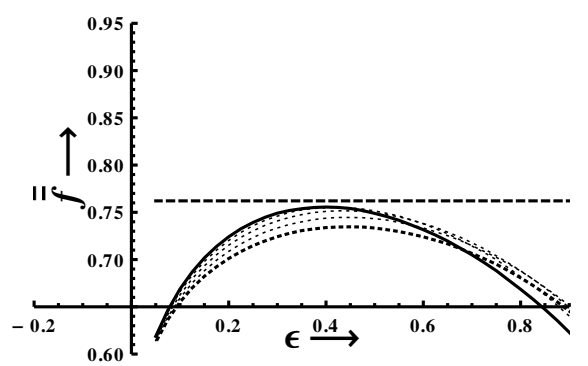

(b)

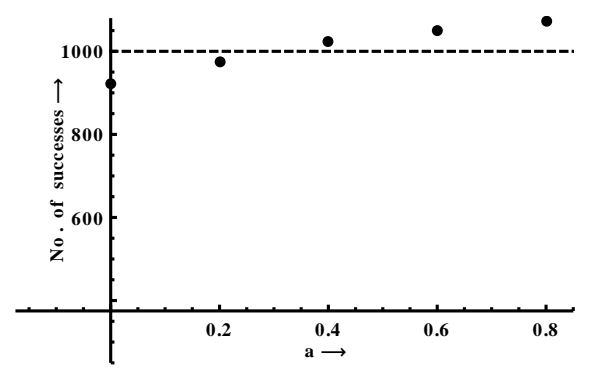

FIG. 2: (a) Plot of the mean fidelity $\overline{\bar{f}}$ for a state with ensemble size 30 and mean calculated over 1000 runs, further averaged over 2000 randomly chosen states, as a function of the coupling strength $\epsilon$. Different curves represent different values of the discard parameter $a$. The discard parameter used are $a=0$ (dotted thick line), $a=0.2$ (dotted line), $a=0.4$ (dotted line) $a=0.6$ (dotted line) and $a=0.8$ (solid line). The straight dotted line represents projective measurements. The solid line comes very close to the projective measurements. (b) Plot of the number of times our schemes outperform the projective measurement based scheme for the 2000 randomly chosen states of the qubit as a function of the discard parameter $a$.

a single qubit, we need to estimate the numbers $(x, y, z)$. The performance of our scheme is quantified using the fidelity measure:

$$
f=1-\left[\left(x-x_{e s t}\right)^{2}+\left(y-y_{e s t}\right)^{2}+\left(z-z_{\text {est }}\right)^{2}\right]
$$

\section{B. Average performance over Bloch sphere}

We move on to test our scheme on a large number of randomly generated states of a qubit and look for the average performance of the scheme over the Bloch sphere. The process is carried out for 2000 states generated randomly. We also study the dependence on ensemble size and use ensemble sizes of 30 and 60 . For each case the simulation is repeated 1000 times to average over statistical fluctuations.

While we average the fidelity over all states to obtain the average fidelity we also keep track whether the scheme outperformed or underperformed as compared to 
(a)

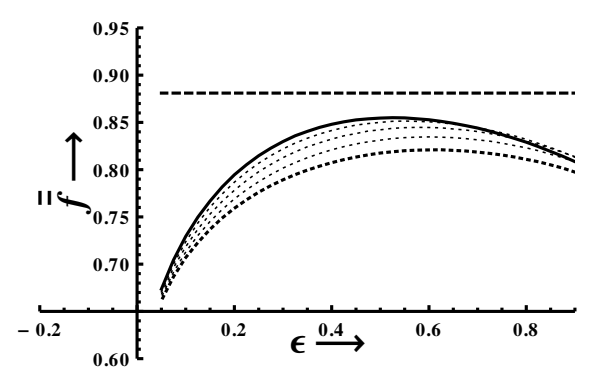

(b)

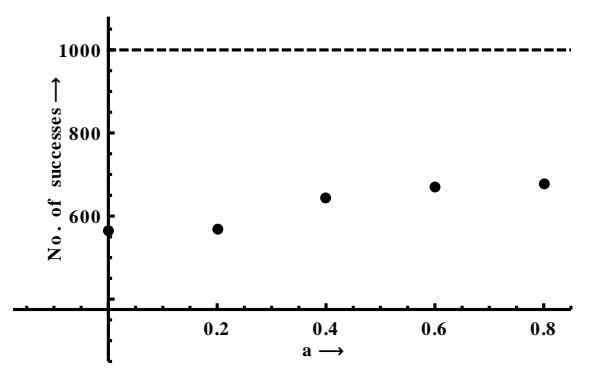

FIG. 3: (a) Plot of the mean fidelity $\overline{\bar{f}}$ for a state with ensemble size 60 and mean calculated over 1000 runs, further averaged over 2000 randomly chosen states, as a function of the coupling strength $\epsilon$. Different curves represent different values of the discard parameter $a$. The discard parameter used are $a=0$ (dotted thick line), $a=0.2$ (dotted line), $a=0.4$ (dotted line) $a=0.6$ (dotted line) and $a=0.8$ (solid line). The straight dotted line represents the projective measurements. (b) Plot of the number of times our schemes outperform the projective measurement based scheme for the 2000 randomly chosen states of the qubit as a function of the discard parameter $a$. The success rate goes down with an increase in ensemble size from 30 to 60 .

the projective measurement scheme in each case. For the ensemble size of 30 , the results of this simulation are presented in two different ways in Figure 2. We calculate the mean fidelities averaged over these states, $\overline{\bar{f}}$, with and without discard, which are then plotted against $\epsilon$ in Figure 2(a). This graph shows an improvement as we increase the amount of discard. We also present our results through a score plot, where we compute the number of states out of 2000 starting states for which our scheme outperforms the projective measurement scheme. The score plot is described in Figure 2(b). Interestingly, this number crosses the $50 \%$ mark for a threshold value of the discard parameter.

When a study of mean fidelity, averaged over 2000 states, $\overline{\bar{f}}$ vs $\epsilon$ was done, it turns out that although on the average the performance of projective measurements is better, if ambiguous meter readings are discarded, then the number of states for which our tomography scheme is successful, goes up. In fact, number of successes out of 2000 for the discard parameter values of $0,0.2,0.4,0.6$ and 0.8 are $923,973,1023,1051$ and 1071, respectively. This we think is a clear evidence that our scheme has the potential of unearthing more information than projective measurements under certain circumstances. In particular, if we are given 30 copies of a unknown state of a qubit, our scheme will be a better choice for carrying out state tomography.

We now turn to testing our scheme with increasing ensemble size. We repeat the simulation in exactly the same way for the case of ensemble size 60 . The results are presented in a similar way in Figure 3 Increasing the ensemble size clearly reduces the efficacy of our scheme as compared to projective measurements. The score plot show that our scheme outperforms the projective measurement scheme for ensemble size of 60 for lesser number of states and the number is less than $50 \%$. Therefore we conclude that our scheme is preferable only when we have a small ensemble size. We would like to clarify that this not due to statistical fluctuations as we have taken the average over a large number of runs even when the ensemble size is small. For a more extensive study of state estimation by this scheme see $\underline{\underline{41}}$.

\section{CONCLUDING REMARKS}

We have presented a scheme for state estimation based on weak measurements. The weak or unsharp measurements that we have used are those where the apparatus system coupling is weak. In this regime, although the information obtained from the system is limited, the corresponding disturbance caused to the state is also small. Thus the possibility of re-using the states becomes available. We show that for small ensemble sizes, the weak measurement based scheme can outperform the projective measurement based scheme. This opens up new possibilities for extracting information from quantum systems.

We have recently extended these results to the domain of continuous variable systems with one degree of freedom. We explore how a weak measurement tomography scheme can be used to estimate the Gaussian state of such systems. We have interesting and encouraging preliminary findings in this context, which will be presented in a forthcoming publication.
* Electronic address: debmalya@iisermohali.ac.in

1 T. A. Brun, American Journal of Physics 70, 719 (2002).

${ }^{2}$ P. Busch and P. J. Lahti, Phys. Rev. D 29, 1634 (1984).
${ }^{3}$ K. Kraus, A. Böhm, J. D. Dollard, and W. Wootters, in States, Effects, and Operations Fundamental Notions of Quantum Theory (1983), vol. 190. 
4 S. T. Ali and G. G. Emch, Journal of Mathematical Physics 15, 176 (1974).

${ }^{5}$ E. Prugovecki, Journal of Mathematical Physics 17, 1673 (1976).

6 S. T. Ali and E. Prugovecki, Journal of Mathematical Physics 18, 219 (1977).

7 L. Disi, Fortschritte der Physik 51, 96 (2003), ISSN 15213978.

8 Y. Aharonov, D. Z. Albert, and L. Vaidman, Phys. Rev. Lett. 60, 1351 (1988).

9 M. A. Nielsen and I. L. Chuang, Quantum computation and quantum information (Cambridge University Press, Cambridge, 2000).

10 A. Peres, Quantum theory: concepts and methods, vol. 57 of Fundamental Theories of Physics (Kluwer Academic Publishers Group, Dordrecht, 1993).

11 S. Massar and S. Popescu, Phys. Rev. Lett. 74, 1259 (1995).

12 M. Ueda and M. Kitagawa, Phys. Rev. Lett. 68, 3424 (1992).

13 C. Branciard, 110, 6742 (2013).

14 Y. W. Cheong and S.-W. Lee, Physical Review Letters 109, 150402 (2012), 1203.4909.

15 N. D. H. Dass, ArXiv e-prints (2014), 1406.0270.

16 L. A. Rozema, A. Darabi, D. H. Mahler, A. Hayat, Y. Soudagar, and A. M. Steinberg, Phys. Rev. Lett. 109, 100404 (2012).

17 O. Oreshkov and T. A. Brun, Phys. Rev. Lett. 95, 110409 (2005).

18 J. S. Lundeen, B. Sutherland, A. Patel, C. Stewart, and C. Bamber, Nature (2011), 1112.3575.

19 J. S. Lundeen and C. Bamber, Phys. Rev. Lett. 108, 070402 (2012).

${ }^{20}$ H. F. Hofmann, ArXiv e-prints (2013), 1311.0093.

21 S. Wu, Scientific Reports 3, 1193 (2013), 1212.3655.

${ }^{22}$ Y. Shikano and S. Tanaka, EPL (Europhysics Letters) 96, 40002 (2011).
${ }^{23}$ H. Kobayashi, K. Nonaka, and Y. Shikano, Phys. Rev. A 89, 053816 (2014).

24 H. F. Hofmann, Phys. Rev. A 81, 012103 (2010).

25 J. V. Neumann, Mathematical foundations of quantum mechanics, 2 (Princeton university press, 1955).

26 I. M. Duck, P. M. Stevenson, and E. C. G. Sudarshan, Phys. Rev. D 40, 2112 (1989).

27 S. Marcovitch and B. Reznik, ArXiv e-prints (2010), 1005.3236

28 M. E. Goggin, M. P. Almeida, M. Barbieri, B. P. Lanyon, J. L. OBrien, A. G. White, and G. J. Pryde (2011).

29 U. Singh and A. K. Pati, Annals of Physics 343, 141 (2014), ISSN 0003-4916.

30 R. Jozsa, Phys. Rev. A 76, 044103 (2007).

31 Y. Aharonov, S. Popescu, and J. Tollaksen, Physics Today 63, 27 (2010).

32 P. B. Dixon, D. J. Starling, A. N. Jordan, and J. C. Howell, Phys. Rev. Lett. 102, 173601 (2009).

33 A. Nishizawa, K. Nakamura, and M.-K. Fujimoto, Phys. Rev. A 85, 062108 (2012).

34 T. Koike and S. Tanaka, Phys. Rev. A 84, 062106 (2011).

35 Y. Aharonov, S. Popescu, D. Rohrlich, and P. Skrzypczyk, New Journal of Physics 15, 113015 (2013).

36 T. Denkmayr, H. Geppert, S. Sponar, H. Lemmel, A. Matzkin, J. Tollaksen, and Y. Hasegawa, Nat Commun 5 (2014).

37 J. Combes, C. Ferrie, Z. Jiang, and C. M. Caves, ArXiv e-prints (2013), 1309.6620.

38 C. Ferrie and J. Combes, Phys. Rev. Lett. 112, 040406 (2014).

39 L. Vaidman, ArXiv e-prints (2014), 1402.0199.

40 J. J. Sakurai, Modern Quantum Mechanics (Revised Edition) (Addison Wesley, 1993), ISBN 0201539292.

41 D. Das and Arvind, Phys. Rev. A 89, 062121 (2014).

42 A more detailed account for the qubit case is available in our recent paper 41 